\title{
Anti-Angiogenesis Effects Induced by Octaminomycins A and B against HUVECs
}

\author{
Jun-Pil Jang $^{1 \dagger}$, Jang Mi Han ${ }^{2 \dagger}$, Hye Jin Jung ${ }^{2 \dagger}$, Hiroyuki Osada ${ }^{3}$, Jae-Hyuk Jang ${ }^{1 *}$, and Jong Seog Ahn ${ }^{1 *}$ \\ ${ }^{1}$ Anticancer Agent Research Center, Korea Research Institute of Bioscience and Biotechnology, Cheongju 28116, Republic of Korea \\ ${ }^{2}$ Department of Pharmaceutical Engineering and Biotechnology, Sun Moon University, Asan 61460, Republic of Korea \\ ${ }^{3}$ Chemical Biology Research Group, RIKEN Center for Sustainable Research Science, 2-1 Hirosawa, Wako, Saitama 351-0198, Japan
}

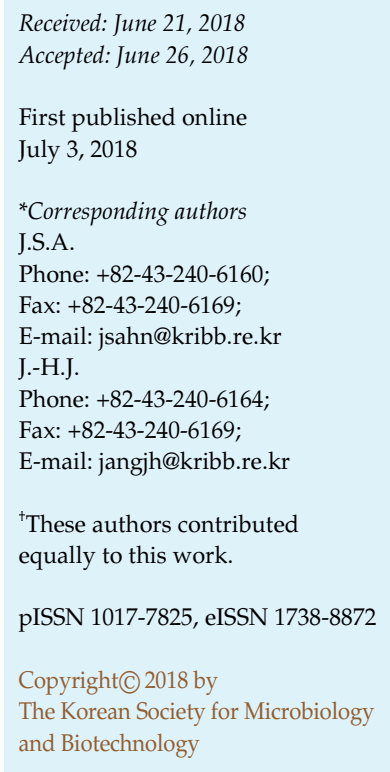

In the course of studies to discover natural products with anti-angiogenic properties, two cyclic octapeptides, octaminomycins A (1) and B (2), were isolated from the cultures of Streptomyces sp. RK85-270. Octaminomycins suppressed the vascular endothelial growth factor (VEGF)-induced proliferation, adhesion, tube formation, migration, and invasion of HUVECs. Anti-angiogenic activity was futher confirmed in vivo by the chicken chorioallantoic membrane assay. We also identified that $\mathbf{1}$ and $\mathbf{2}$ inhibited the phosphorylation of VEGF receptor 2, AKT, and ERK1/2 and the expression and activities of MMP-2 and MMP-9. These results suggest that $\mathbf{1}$ and $\mathbf{2}$ may serve as potential scaffolds for the development of therapeutic agents to angiogenesis-dependent diseases.

Keywords: Octaminomycin, anti-angiogenesis, HUVEC, VEGF, secondary metabolites

\section{Introduction}

Microbial secondary metabolites are widely considered to be one of the best resources for drug discovery and research reagents [1, 2]. Among them, actinomycetes are important sources in the production of hit and lead compounds for pharmacological applications [3]. Approximately $40 \%$ of microbial bioactive compounds are derived from actinobacteria [4]. These secondary metabolites are also important chemical probes, which are chemical tools used to investigate biological functions [5].

Angiogenesis is the generation of new blood vessels from existing vessels. This phenomenon involves several steps such as migration, proliferation, and formation of capillary tubes in endothelial cells $[6,7]$ and has an important role during organ development, as well as in wound healing processes. However, excessive angiogenesis occurs cancer spread and growth, macular degeneration, and chronic inflammatory diseases. The family of vascular endothelial growth factor (VEGF) and related receptors (VEGFRs) play a critical role in the regulation of pathological angiogenesis $[8,9]$. VEGF has been shown to strongly induce proliferation, cell migration, and tube formation with a chracteristic specificity for endothelial cells [10]. Therefore, angiogenesis inhibition via VEGF-mediated crucial signaling has been considered a promising strategy for the treatment of angiogenesis-related diseases.

For many decades, angiogenesis inhibitors have been developed to reduce the progression and invasiveness of many tumors. However, clinical studies showed that toxicity and resistance are the primary challenges confronting the development of ideal angiogenesis inhibitors [11]. In our continuing efforts to search microbial bioactive secondary metabolites with an anti-angiogenic efficacy, two cyclic octapeptides, octaminomycins A (1) and B (2), were isolated from Streptomyces sp. RK85-270 (Fig. 1). Here, we 


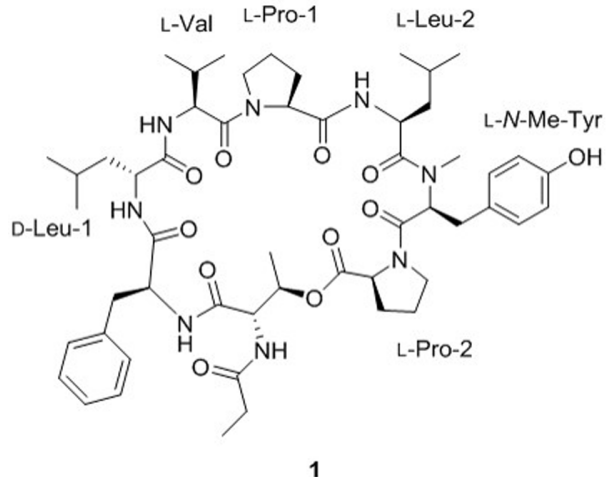

Fig. 1. The structures of octaminomycins A (1) and B (2).

report the effects of compounds $\mathbf{1}$ and $\mathbf{2}$ on anti-angiogenesis in human umbilical vein endothelial cells (HUVECs).

\section{Materials and Methods}

\section{Microbial Isolation and Identification}

The strain RK85-270 was isolated from a soil sample collected at Java, Indonesia. Phylogenetic analysis based on 16S rRNA gene sequence (GenBank Accession No. KY039979) comparisons showed match with Streptomyces endus NRRL 2339 (100\%) and Streptomyces sporocinereus NBRC 100766 (100\%), identifying and designating as Streptomyces sp. RK85-270 [12].

\section{Culture Condition}

Streptomyces sp. RK85-270 was cultured in a 250-ml Erlenmeyer flask containing $50 \mathrm{ml}$ of seed culture medium for 3 days at $28^{\circ} \mathrm{C}$ on a rotary shaker with agitation at $125 \mathrm{rpm}$. For a large culture, $1 \%$ of the pre-culture broth was inoculated into $40 \times 1,000 \mathrm{ml}$ baffled Erlenmeyer flasks containing $250 \mathrm{ml}$ of modified CDY broth, which were cultured for 8 days at $28^{\circ} \mathrm{C}$ on a rotary shaker with agitation at $125 \mathrm{rpm}[12]$.

\section{Isolation of Octaminomycins A (1) and B (2)}

The crude extract was fractionated by reversed phase $C_{18}$ vaccum column chromatography with a stepwise solvent system of $\mathrm{MeOH}: \mathrm{H}_{2} \mathrm{O}$ (20:80 to 100:0, each $\left.\times 1 \mathrm{l}\right)$. The $70 \%(43.4 \mathrm{mg})$ fraction was further separated by RP-HPLC with $\mathrm{CH}_{3} \mathrm{CN}-\mathrm{H}_{2} \mathrm{O}$ (50:50) to give octaminomycins A (1, $\left.13 \mathrm{mg}, t_{\mathrm{R}} 34.9 \mathrm{~min}\right)$ and $\mathrm{B}(\mathbf{2}$, $\left.40 \mathrm{mg}, t_{\mathrm{R}} 45.5 \mathrm{~min}\right)$.

\section{Materials}

1 and 2 were prepared at a concentration of $10 \mathrm{mM}$ using dimethyl sulfoxide (DMSO). Fetal bovine serum (FBS) was from Invitrogen (USA), and Endothelial growth medium-2 (EGM-2) and antibiotics were from Lonza (USA). Transwell chamber, laminin, recombinant human VEGF, and Matrigel were from Corning Costar (USA), Bio Lamina (Sweden), Koma Biotech

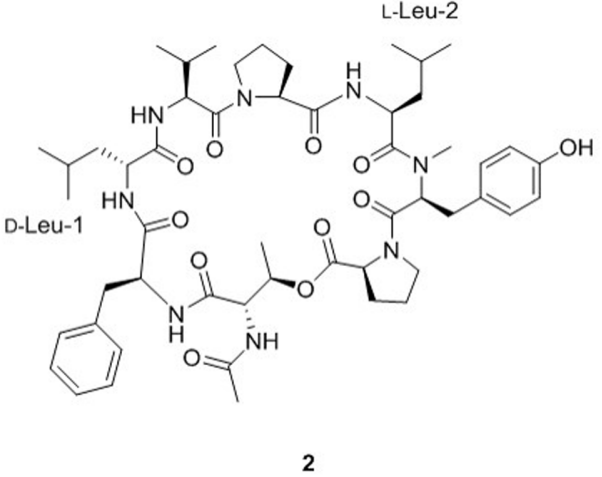

(Korea), and BD Biosciences (USA), respectively. MG132 and gelatin were from Sigma-Aldrich (USA). VEGFR2, phosphoVEGFR2 (Tyr1175), AKT, phospho-AKT (Ser473), ERK1/2, phospho-ERK1/2 (Thr202/Tyr204), MMP-2, MMP-9, and $\beta$-actin antibodies were from Cell Signaling Technology (USA).

\section{HUVEC Culture}

Human umbilical vein endothelial cells (HUVECs) were maintained using EGM-2 supplemented with 10\% FBS and 1\% antibiotics in a humidified $37^{\circ} \mathrm{C} / 5 \% \mathrm{CO}_{2}$ incubator.

\section{Cell Proliferation Assay}

Cell proliferation was measured using the 3-(4,5-dimethylthiazol2-yl)-2,5-diphenyltetrazolium bromide (MTT) colorimetric assay. HUVECs were seeded in a 96-well culture plate at a density of $3 \times 10^{3}$ cells/well and then treated with various concentrations of 1 and 2 in the presence or absence of VEGF for $72 \mathrm{~h}$. After incubation, $50 \mu \mathrm{l}$ of MTT solution ( $2 \mathrm{mg} / \mathrm{ml}$; Sigma-Aldrich) was added to each well followed by incubation for $3 \mathrm{~h}$ at $37^{\circ} \mathrm{C}$, and then the culture medium was removed, and DMSO was added to each well. The absorbance of each well was detected at $540 \mathrm{~nm}$ using a microplate spectrophotometer (Thermo Fisher Scientific, Vantaa, Finland).

\section{Migration Assay}

Wound healing assay was assessed to observe cell migration. HUVECs $\left(1 \times 10^{5}\right.$ cells/well) were seeded on a 24 -well culture plate and scratched using a sterile tip. Each well was washed with EGM-2 supplemented with $1 \%$ FBS, and the cells were treated with 1 and 2 in the presence or absence of VEGF. After $24 \mathrm{~h}$, photographs were taken under a microscope (Olympus, USA).

\section{Adhesion Assay}

The cell-matrix adhesion assay was performed in a 24-well culture plate coated with laminin overnight at $4^{\circ} \mathrm{C}$. HUVECs $\left(5 \times 10^{4}\right.$ cells/well) were cultured to each well in EGM-2 containing $1 \%$ FBS and treated with $\mathbf{1}$ and $\mathbf{2}$ in the presence or absence of VEGF. After $2 \mathrm{~h}$, unbound cells were carefully removed, and 
attached cells were observed and counted under a microscope (Olympus).

\section{Chemoinvasion Assay}

Cell invasion was assessed using a Transwell membrane $(8-\mu \mathrm{m}$

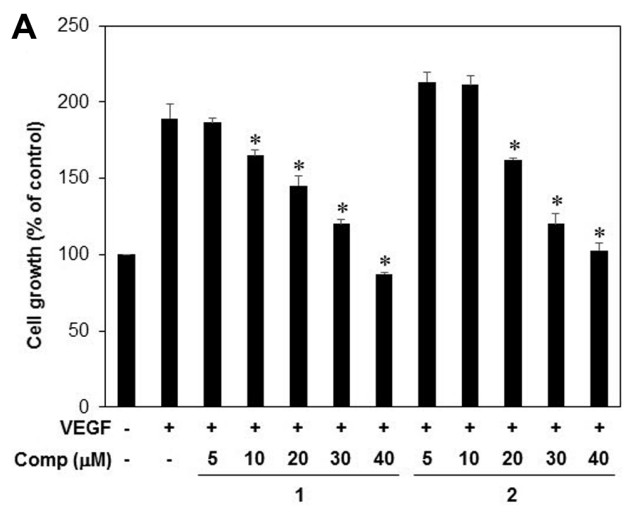

B
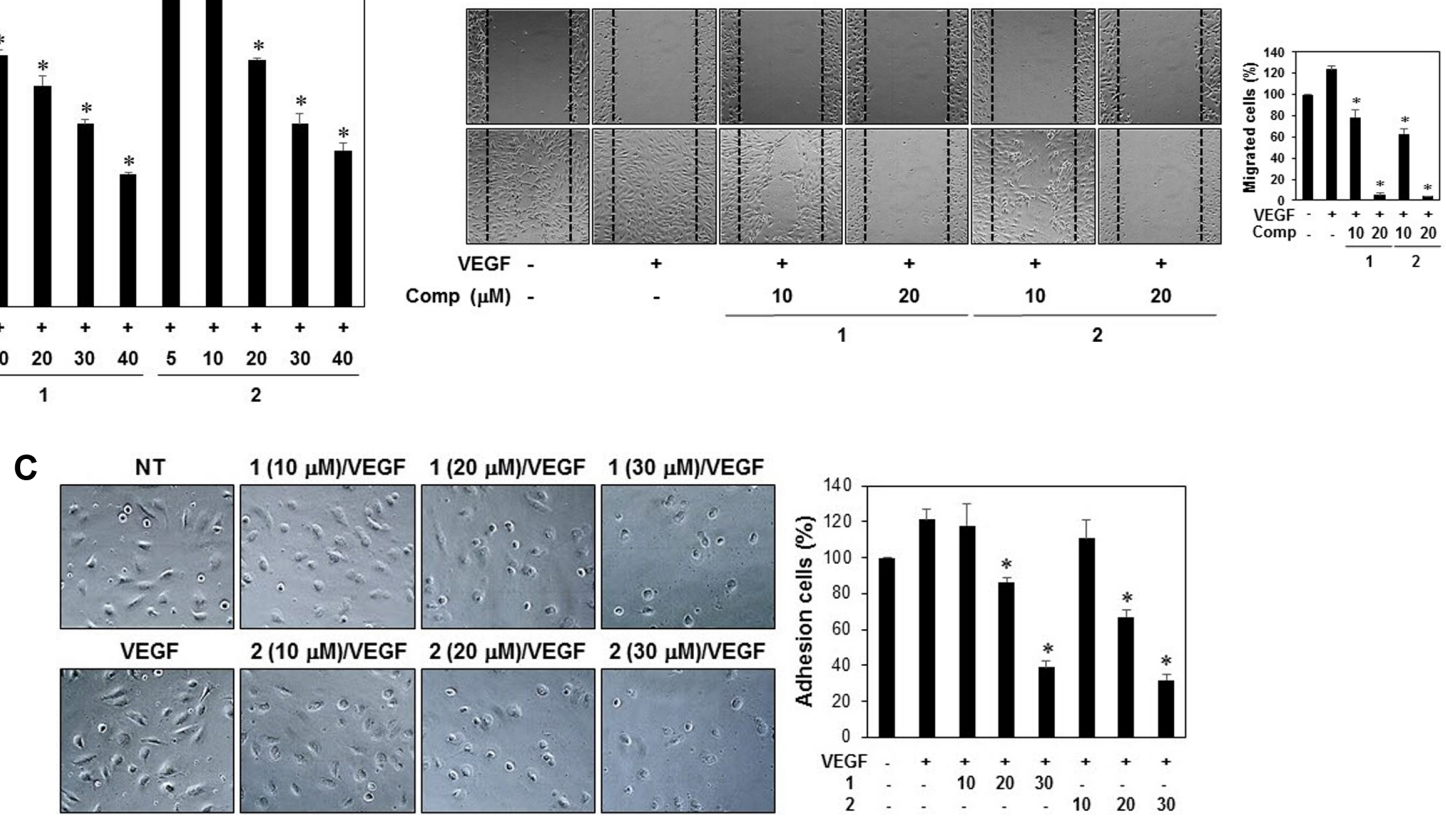

D
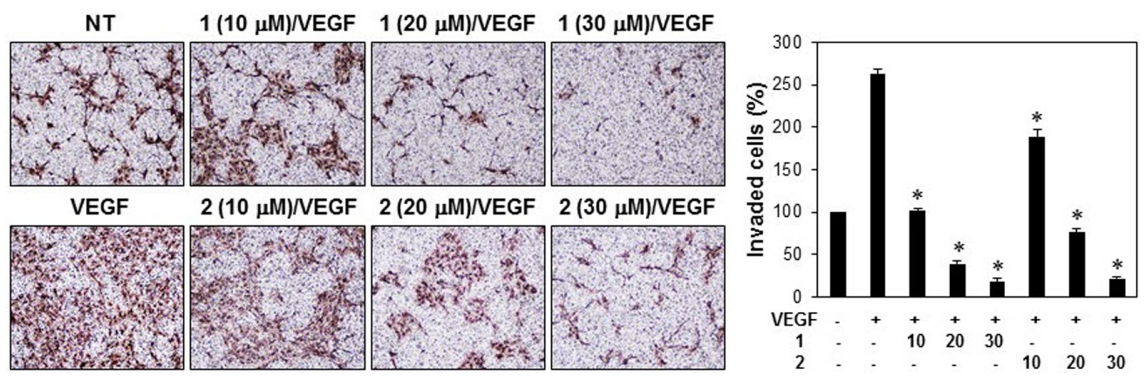

E
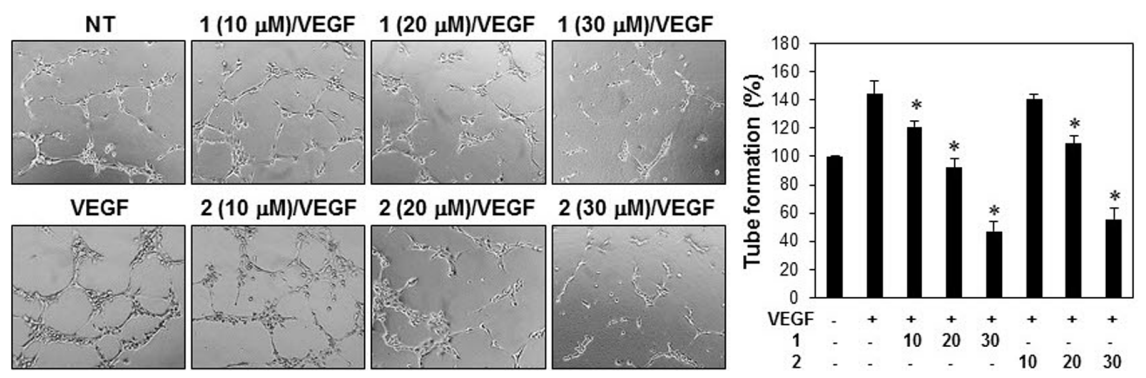

Fig. 2. The in vitro anti-angiogenic activities of octaminomycins A (1) and B (2).

Serum-starved HUVECs were stimulated with VEGF $(30 \mathrm{ng} / \mathrm{ml})$ in the presence or absense of 1 and 2. (A) The effects of 1 and 2 on the proliferation of HUVECs. The growth of HUVECs was determined after $72 \mathrm{~h}$ using an MTT colorimetric assay. (B) The effects of $\mathbf{1}$ and $\mathbf{2}$ on the migration of HUVECs. The closure of the wound was visualized after $24 \mathrm{~h}$ under a microscope. (C) The effects of $\mathbf{1}$ and 2 on the adhesion of HUVECs. The attached cells to laminin after $2 \mathrm{~h}$ were counted under a microscope. (D) The effects of $\mathbf{1}$ and $\mathbf{2}$ on the invasion of HUVECs. The invaded cells after $24 \mathrm{~h}$ were stained with hematoxylin/eosin and visualized under a microscope. (E) The effects of $\mathbf{1}$ and $\mathbf{2}$ on the tube-forming ability of HUVECs. The formed tubes after $6 \mathrm{~h}$ were visualized under a microscope. ${ }^{*} p<0.05$ versus the VEGF control. 
pore size) according to manufacturer's instruction. Briefly, the lower side of the filter was coated with $10 \mu \mathrm{l}$ of gelatin $(1 \mathrm{mg} / \mathrm{ml})$, and the upper side was coated with $10 \mu \mathrm{l}$ of Matrigel $(3 \mathrm{mg} / \mathrm{ml})$. Serum-starved HUVECs were seeded at $8 \times 10^{4}$ cells/well onto upper chamber of Transwell plate, and $\mathbf{1}$ and $\mathbf{2}$ were added to each lower chamber well in the presence or absence of VEGF. Following incubation for $24 \mathrm{~h}$, the cells were fixed with methanol and stained with hematoxylin and eosin (H\&E). The number of invaded cells was counted under an optical microscope (Olympus).

\section{Tube Formation Assay}

The capillary-like tube formation was performed on Matrigel. A 48-well culture plate was coated with Matrigel, and HUVECs $\left(3 \times 10^{4}\right.$ cells/well) were plated in the coated wells. 1 and 2 were then added to each well in the presence or absence of VEGF. After $6 \mathrm{~h}$, formed tube was observed under a microscope (Olympus).

\section{Chorioallantoic Membrane (CAM) Assay}

Fertilized chicken eggs were maintained at $37^{\circ} \mathrm{C}$ for 3 days in an egg incubator. To separate the chicken CAM from the shell membrane, egg albumin was taken out with a hypodermic syringe. After 2 days, the shell was peeled away to expose CAM. The coverslips pretreated with vehicle alone, $\mathbf{1}$ or $\mathbf{2}$ were put on the CAM. After incubation for 2 days, the formed blood vessels were observed by injecting 10\% fat emulsion (Sigma-Aldrich) into the CAM and photographed under a microscope.

\section{Western Blotting}

The cell lysate samples were applied to SDS-PAGE and the separated proteins were then transferred onto PVDF membranes (Millipore, USA). The blots were blocked with 5\% skim milk at room temperature for $1 \mathrm{~h}$, and then incubated at $4^{\circ} \mathrm{C}$ overnight with the primary antibodies. Immunolabeling was detected with an enhanced chemiluminescence (ECL) kit (Bio-Rad Laboratories, USA), according to the manufacturer's instructions.

\section{Gelatin Zymography}

Gelatin zymography was performed to detect the proteolytic activities of MMP-2 and MMP-9. Conditioned media from cell cultures were mixed with 5X SDS sample buffer and loaded onto a 10\% gelatin Zymogram-PAG 10\% Pre-cast gel (Komabiotech, Korea). To renature the enzymes, the gel was incubated twice for $20 \mathrm{~min}$ in renaturation buffer (Komabiotech) and incubated in Zymogram development buffer (Komabiotech) for $20 \mathrm{~min}$, and then placed in fresh Zymogram development buffer at $37^{\circ} \mathrm{C}$ for $36 \mathrm{~h}$. The gel was stained with Coomassie Brilliant Blue R-250 (Komabiotech), and then destained with destaining solution. The presence of clear bands in the gel at the appropriate molecular weights reflects proteolytic activities of MMP-2 and MMP-9.

\section{Statistical Analysis}

The results are expressed as the mean \pm standard error (SE). Student's $t$ test was used to decide statistical significance between the control and test groups. The $p$ value of $<0.05$ is judged statistically significant.

\section{Results}

Angiogenesis depends on the proliferation, migration, proteolytic degradation of the extracellular matrix (ECM) and the capillary tube formation of endothelial cells, and VEGF is a major pro-angiogenic factor that mediates these processes [13,14]. To explore the anti-angiogenic activities of 1 and 2, we evaluated the activities of 1 and 2 on the growth of HUVECs stimulated by VEGF using the MTT colorimetric assay. As shown in Fig. 2A, 1 and 2 dosedependently inhibited the VEGF-induced proliferation of HUVECs. The inhibitory activities of $\mathbf{1}$ and $\mathbf{2}$ on the migration of HUVECs were evaluated using a wound healing assay. The treatment with $\mathbf{1}$ and $\mathbf{2}$ remarkably suppressed the endothelial cell migration even in the presence of VEGF (Fig. 2B). In addition, the cell adhesion of VEGF-induced HUVECs to the laminin, an ECM protein, was inhibited by treatment with $\mathbf{1}$ and $\mathbf{2}$ in a dosedependent manner (Fig. 2C). To further assess the antiangiogenic potential of $\mathbf{1}$ and $\mathbf{2}$, a Matrigel invasion assay was performed, and treatment with compounds 1 and 2 resulted in a significant reduction in the invasiveness of the HUVECs mediated by VEGF (Fig. 2D). We next tested the activities of $\mathbf{1}$ and $\mathbf{2}$ on the VEGF-induced formation of

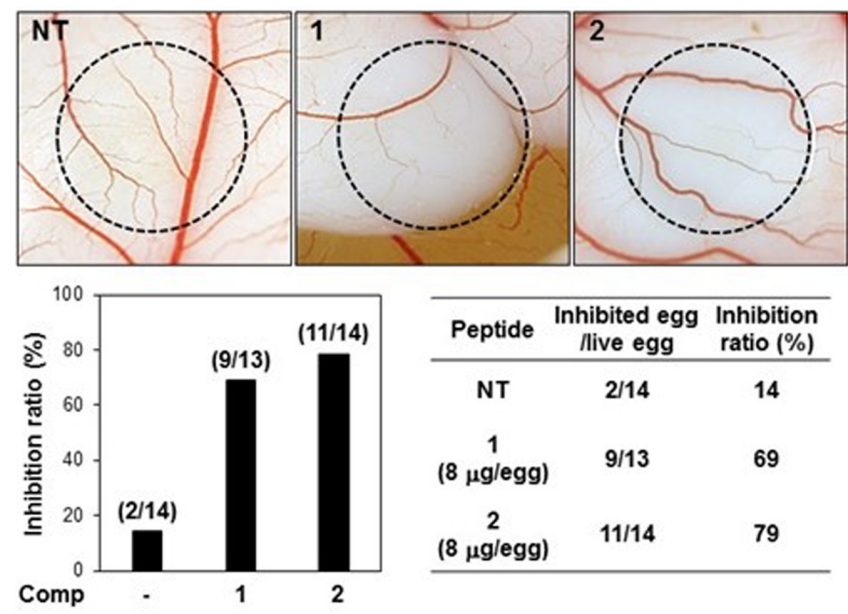

Fig. 3. The in vivo antiangiogenic activities of octaminomycins A (1) and B (2).

Fertilized chick eggs were maintained in a humidified incubator at $37^{\circ} \mathrm{C}$. At embryonic day 4.5, coverslips loaded with vehicle alone, 1 or 2 were placed to the CAM surface. Two days later, the chorioallantois was observed under a microscope. Calculations were based on the ratio of inhibited eggs relative to the total number of live eggs. 
capillary-like structures by the HUVECs. Treatment with VEGF alone stimulated the robust tubular capillary structures on the Matrigel, and $\mathbf{1}$ and $\mathbf{2}$ effectively decreased the VEGF-induced tube formation of the HUVECs in a dosedependent manner. (Fig. 2E). These results demonstrate that 1 and 2 potently inhibit the VEGF-induced angiogenesis of HUVECs in vitro.

The chicken chorioallantoic membrane (CAM) assay has been generally used for the in vivo validation of the angiogenesis inhibitory potential of bioactive compounds [13]. To verify the anti-angiogenic activities of $\mathbf{1}$ and $\mathbf{2}$ in vivo, the CAM assay was done. Coverslips containing 1 and 2 were placed on the CAM surface, and small tortuous blood vessels, a characteristic aspect of neovascularization, were observed under a microscope. As shown in Fig. 3, the inhibition of neovascularization on the control coverslips was $14 \%(n=14)$, whereas 1 and 2 much more significantly inhibited the angiogenesis of the CAM $(69 \%$ at $8 \mu \mathrm{g} / \mathrm{egg}$ of octaminomycin A, $n=13 ; 79 \%$ at $8 \mu \mathrm{g} / \mathrm{egg}$ of octaminomycin $\mathrm{B}, n=14)$ without toxicity against pre-existing vessels. These data indicate that 1 and 2 exhibit promising antiangiogenic activities both in vitro and in vivo.

It is well known that the VEGFR2 signaling pathway has a vital role in angiogenesis [15]. To further elucidate the underlying mechanisms of the anti-angiogenic effects of 1 and 2, we evaluated several key signaling molecules involved in the VEGFR2-mediated signaling pathway in HUVECs. As shown in Fig. 4A, 1 and 2 efficiently
A

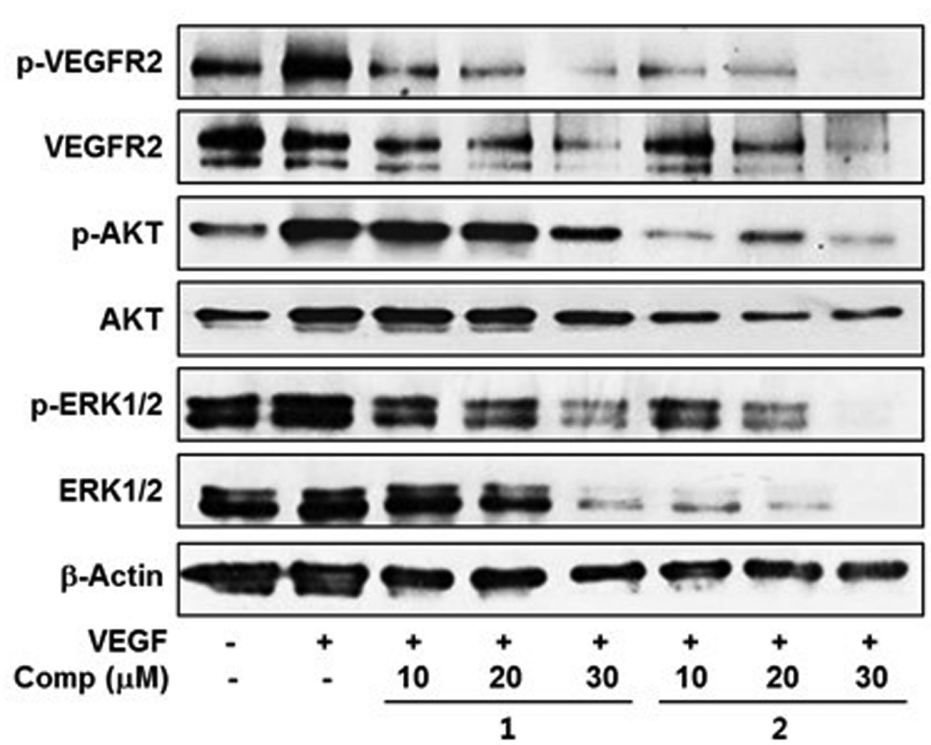

B

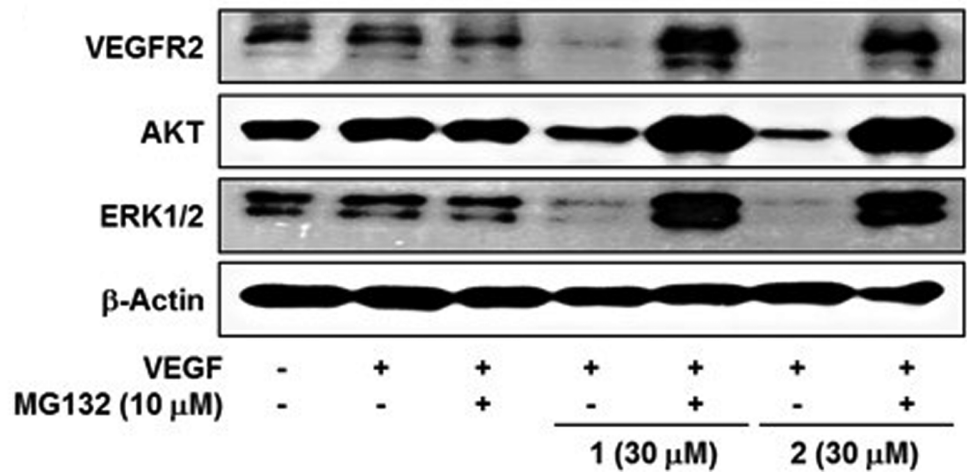

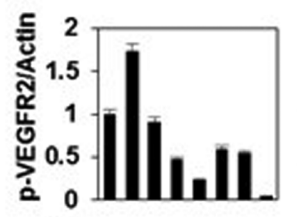
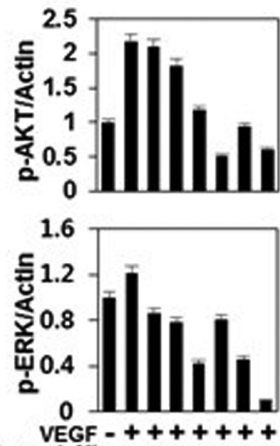

Comp $(\mu M)=-102030102030$ $\frac{1}{2}$
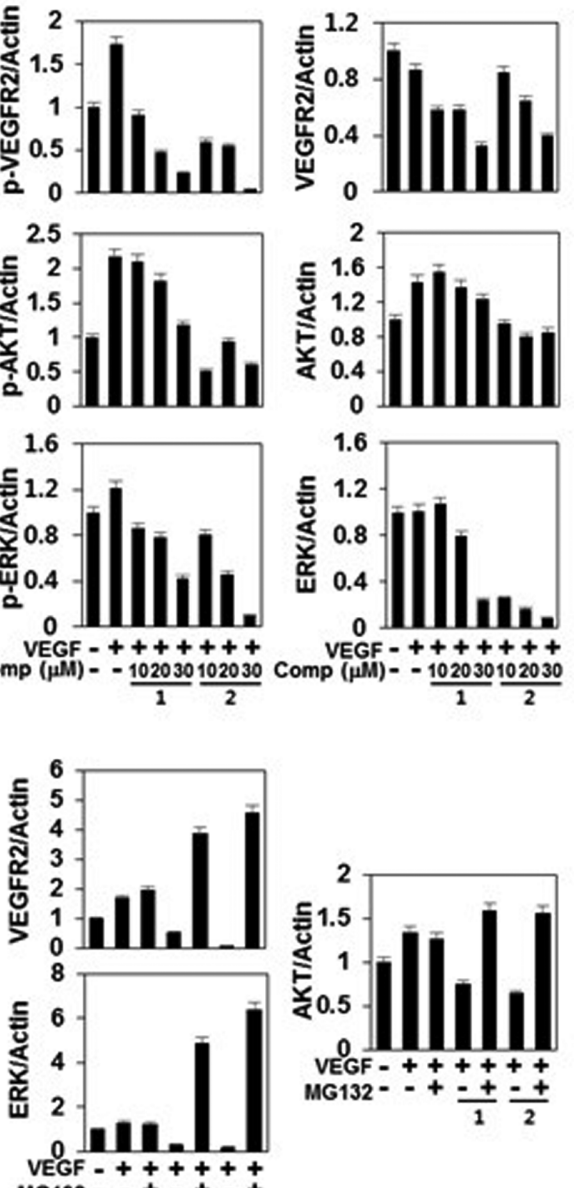

MG132:- $+ \pm+ \pm+t$

Fig. 4. The effects of octaminomycins A (1) and B (2) on the VEGFR2 signal transduction.

Serum-starved HUVECs were pretreated with 1, 2 and/or MG132 $(10 \mu \mathrm{M})$ for $3 \mathrm{~h}$ and then stimulated with VEGF ( $30 \mathrm{ng} / \mathrm{ml})$ for $5 \mathrm{~min}$. Protein levels were detected by Western blot analysis using specific antibodies and further quantified by densitometry. The level of $\beta$-actin was used as an internal control. (A) The effects of $\mathbf{1}$ and $\mathbf{2}$ on the VEGFR2 signaling in HUVECs. (B) The effect of MG132 on the protein levels of VEGFR2, AKT and ERK1/2 inhibited by 1 and 2 . 
suppressed the phosphorylation of VEGFR2, AKT, and ERK1/2 induced by VEGF but also inhibited the total protein levels of VEGFR2, AKT, and ERK1/2. To assess whether $\mathbf{1}$ and $\mathbf{2}$ affect the proteasomal degradation of VEGFR2, AKT, and ERK1/2 proteins, we investigated the inhibitory effect of a proteasome inhibitor MG132 on the degradation of the proteins by the activities of $\mathbf{1}$ and $\mathbf{2}$. As shown in Fig. 4B, treatment with MG132 abolished the degradation of VEGFR2, AKT, and ERK1/2 proteins by 1 and $\mathbf{2}$, indicating that $\mathbf{1}$ and $\mathbf{2}$ induce the proteolysis of VEGFR2-mediated downstream effectors in HUVECs. These data suggest that $\mathbf{1}$ and $\mathbf{2}$ may show the anti-angiogenic activities by inhibiting VEGFR2-mediated downstream signaling cascades by decreasing the protein stability of these signaling molecules.

Angiogenesis requires the matrix metalloproteinase (MMP)-mediated degradation of the blood vessel basement membrane which contains various ECM proteins [16]. We examined whether $\mathbf{1}$ and $\mathbf{2}$ affect the MMP protein expression and activity in HUVECs. As shown in Fig. 5, 1 and 2 reduced not only the protein expression levels, but also the enzymatic activities of MMP-2 and MMP-9, indicating that the anti-angiogenic effects of 1 and 2 may be partly associated with the downregulation of the MMP protein expression and activity.

\section{Discussion}

Two cyclic octapeptides, octaminomycins A (1) and B (2) were isolated from Streptomyces sp. RK85-270 as the promising anti-angiogenic bioactive molecules. Recently, octaminomycins A (1) and B (2) have been reported as antiplasmodial agents $\mathbf{1}$ and $\mathbf{2}$ showed no cytotoxicity against human cervical cancer cells (HeLa), human promyelocytic leukemia cells (HL-60), mouse temperature-sensitive cdc2 mutant cells (tsFT210), and rat kidney cells that were infected with ts 25 ( $s r c^{\text {ts }}$-NRK) [12]. In this study, we found that 1 and $\mathbf{2}$ exhibits anti-angiogenic efficacy both in vitro and in vivo with no cytotoxicity. The anti-angiogenic effects of $\mathbf{1}$ and $\mathbf{2}$ in HUVECs were observed in this study for the first time

In conclusion, we showed that octaminomycins A (1) and B (2) suppress multiple processes in HUVECs including proliferation, migration, adhesion, invasion and tube formation, as well as the neovascularization of the chorioallantoic membrane in developing chick embryos. We also found that $\mathbf{1}$ and $\mathbf{2}$ inhibit the VEGFR2-mediated signaling pathway and lead to a decrease in the expression and activities of MMP-2 and MMP-9. Based on these findings, we suggest that octaminomycins A (1) and B (2) may serve as potential scaffolds for the development of
A
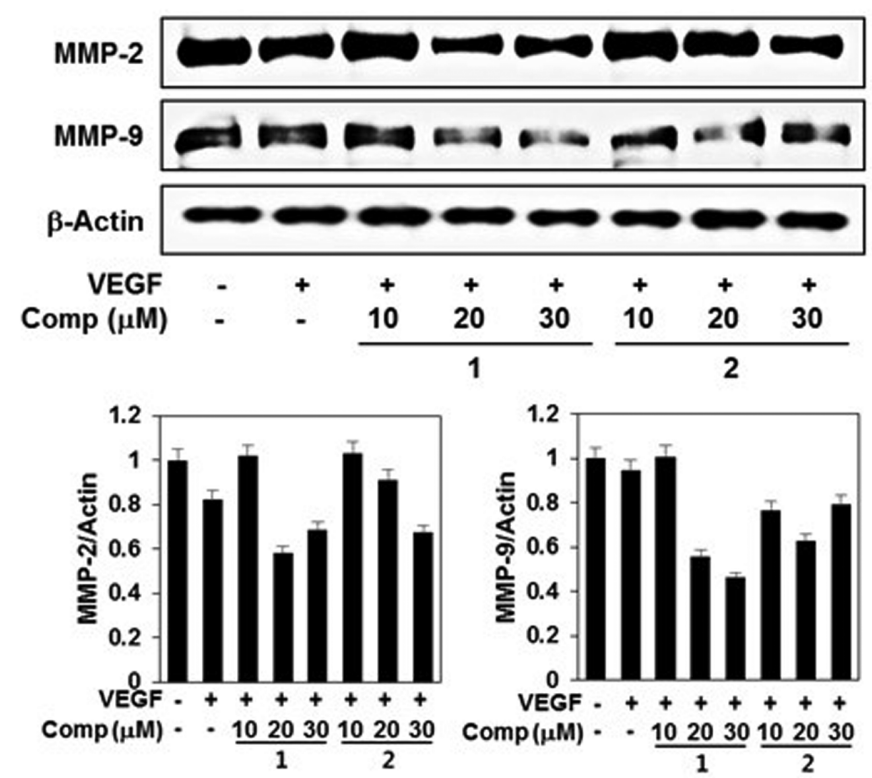

B
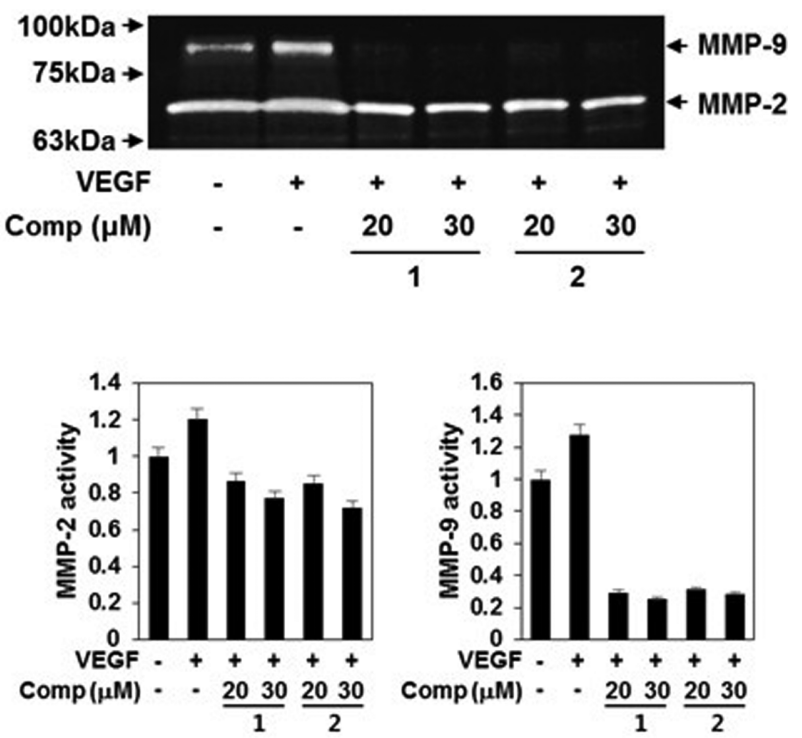

Fig. 5. The effects of octaminomycins A (1) and B (2) on the protein expression and proteolytic activities of MMPs.

Serum-starved HUVECs were treated with $\mathbf{1}$ and $\mathbf{2}$ in the presence or absense of VEGF ( $30 \mathrm{ng} / \mathrm{ml}$ ) for $24 \mathrm{~h}$. (A) Protein levels were determined by Western blotting using specific antibodies and further quantified by densitometry. The level of $\beta$-actin was used as an internal control. (B) Enzymatic activities of MMPs were detected by gelatin zymogram analysis and further quantified by densitometry. 
therapeutic agents to treat angiogenesis-dependent diseases.

\section{Acknowledgments}

This work was supported by the International Joint Research Project (ASIA-16-011) of the NST (National Research Council of Science \& Technology) and KRIBB Research Initiative Program funded by the Ministry of Science ICT (MSIT) of Republic of Korea. This research was also supported by Basic Science Research Program through the National Research Foundation of Korea (NRF) funded by the Ministry of Education (NRF-2016R1D1A1B03932956). We acknowledge the Korea Basic Science Institute, Ochang, South Korea, for providing NMR (700, 800, and $900 \mathrm{MHz}$ ) and HRESIMS data.

\section{Conflict of Interest}

The authors have no financial conflicts of interest to declare.

\section{References}

1. Newman DJ, Cragg GM. 2016. Natural products as sources of new drugs from 1981 to 2014. J. Nat. Prod. 79: 629-661.

2. Cragg GM, Newman DJ. 2013. Natural products: a continuing source of novel drug leads. Biochim. Biophys. Acta 1830: 3670-3695.

3. Clardy J, Fischbach MA, Walsh CT. 2006. New antibiotics from bacterial natural products. Nat. Biotechnol. 24: 1541-1550.

4. Genilloud O, González I, Salazar O, Martín J, Tormo JR, Vicente F. 2011. Current approaches to exploit actinomycetes as a source of novel natural products. J. Ind. Microbiol. Biotechnol. 38: 375-359.

5. Nett M, Ikeda H, Moore BS. 2009. Genomic basis for natural product biosynthetic diversity in the actinomycetes. Nat. Prod. Rep. 26: 1362-1384.

6. Kerbel R, Folkman J. 2002. Clinical translation of angiogenesis inhibitors. Nat. Rev. Cancer 2: 727-739.

7. Jubb AM, Oates AJ, Holden S, Koeppen H. 2006. Predicting benefit from anti-angiogenic agents in malignancy. Nat. Rev. Cancer 6: 626-635.

8. Carmeliet P. 2005. VEGF as a key mediator of angiogenesis in cancer. Oncology 69: 4-10.

9. Ferrara N. 2004. Vascular endothelial growth factor as a target for anticancer therapy. Oncologist 9: 2-10.

10. Ferrara N, Davis-Smyth T. 1997. The biology of vascular endothelial growth factor. Endocr. Rev. 18: 4-25.

11. Berqurs G, Hanahan D. 2008. Modes of resistance to antiangiogenic therapy. Nat. Rev. Cancer 8: 592-603.

12. Jang JP, Nogawa T, Futamura $Y$, Shimizu T, Hashizume D, Takahashi S, et al. 2017. Octaminomycins A and B, cyclic octadepsipeptides active against Plasmodium falciparum. J. Nat. Prod. 27: 134.

13. Staton CA, Reed MW, Brown NJ. 2009. A critical analysis of current in vitro and in vivo angiogenesis assays. Int. J. Exp. Pathol. 90: 195-221.

14. Hoeben A, Landuyt B, Highley MS, Wildiers H, Van Oosterom AT, De Bruijn EA. 2004. Vascular endothelial growth factor and angiogenesis. Pharmacol. Rev. 56: 549-580.

15. Olsson AK, Dimberg A, Kreuger J, Claesson-Welsh L. 2006. VEGF receptor signalling - in control of vascular function. Nat. Rev. Mol. Cell. Biol. 7: 359-371.

16. Hass TL. 2005. Endothelial cell regulation of matrix metalloproteinases. Can. J. Physiol. Pharmacol. 83: 1-7. 